Textures and Microstructures, Vol. 31, pp. 249-261 Reprints available directly from the publisher Photocopying permitted by license only
(C) 1999 OPA (Overseas Publishers Association) N.V.

Published by license under the Gordon and Breach Science Publishers imprint. Printed in Malaysia.

\title{
EFFECT OF MACHINING VELOCITY ON THE CRYSTALLOGRAPHIC TEXTURES IN A DIAMOND TURNED ALUMINIUM SINGLE CRYSTAL
}

\author{
S. TO ${ }^{\mathrm{a}}$, W.B. LEE $^{\mathrm{a}, *}$ and C.Y. CHAN ${ }^{\mathrm{b}}$ \\ ${ }^{a}$ Ultraprecision Machining Centre, Department of \\ Manufacturing Engineering, The Hong Kong Polytechnic University, \\ Kowloon, Hong Kong, China; ${ }^{b}$ Department of \\ Manufacturing Engineering, Hong Kong Technical College, China
}

(Received 20 March 1999)

\begin{abstract}
The orientation changes in the crystallographic textures of a diamond turned aluminium single crystal have been investigated. The $\mathrm{X}$-ray pole figures were collected at various locations on the surfaces turned at high speed. In the central part of the turned surfaces, the pole figures revealed the presence of a thin deformed layer. Four sets of slip systems were found to operate to a very similar extent. However, as the distance from the centre increased, the operation of these four sets of slip systems varied and the textural changes were found to be increasingly affected by the cutting velocity. In a separate grooving experiment, electron back-scatter diffraction (EBSD) patterns were collected at various locations along the bottom part of the groove. These patterns revealed a lattice rotation on the machined surface which was induced by shearing along the cutting direction.
\end{abstract}

Keywords: Aluminium single crystal; Machining; Lattice rotation; Pole figures; EBSD

\section{INTRODUCTION}

Chip formation in metal cutting involves large plastic deformation which in turn produces lattice rotation in the surface region and subsurface. The properties of the free surface and the sub-surface of the machined surface were known to be very dependent on the machining method, the machining parameters together with the physical state of the

\footnotetext{
* Corresponding author.
} 
workpiece materials. Machining processes produce distinguished footprints on the machined surfaces characterised by features like surface roughness, crystallographic textures, mobile dislocation density and twin density.

The change of the near surface texture after face milling and flat grinding had been investigated by other researchers (Krause and Öcalan, 1984; Maurer et al., 1988) and asymmetric textures were reported. The textural changes of face milling and flat grinding were observed to be correlated to those of cold rolling and of hot rolling respectively. However, not much work has been done on the effect of the single point diamond turning on the surface texture (Lee et al., 1996). In ultraprecision diamond turning, the depth of cut is often smaller than the grain size of a polycrystalline materials. Thus, cutting is performed within each individual crystals. The cutting behaviour and mechanism of chip formation are known to be dependent on the crystallographic factors, such as crystal orientation, the slip systems and the mobile dislocation density of the workpiece. The effect of crystal orientation on the cutting mechanism of single crystal has received considerable research interest (König and Senrath, 1991; Ueda and Iwata, 1980; Yuan et al., 1992; 1994; Moriwaki et al., 1993). A physical model based on plane-strain deformation to predict the periodic variation of cutting force and shear angle with cutting direction has been proposed by Lee (1990) and Lee and Zhou (1993) and the theoretical prediction was found to be compatible with the experimental observations.

In this paper, the effect of cutting speed on the variation of surface texture and lattice rotation of diamond turned surface were investigated. $\{111$ pole figures were determined at various locations by $\mathrm{X}$-ray diffraction method. The lattice rotation was also observed at various locations on a machined groove by electron back-scatter diffraction (EBSD) method.

\section{PART 1. X-RAY TEXTURE STUDIES}

\subsection{Experimental Procedure}

The diamond turning experiments were carried out on a two axis CNC contouring lathe from Taylor Hobson Pneumo (Optoform 30). 
The workpiece material in the experiment was a cylindrical aluminium single crystal of which the purity is $99.99 \%$ with the cylindrical axis parallel to $\langle 001\rangle$. The diameter of the workpiece was $12.7 \mathrm{~mm}$ and the length $15 \mathrm{~mm}$. A natural single point diamond tool was used with a cutting tool radius of $0.635 \mathrm{~mm}$, a rake angle of $0^{\circ}$ and a clearance angle of $5^{\circ}$. All the turning processes were carried out at a spindle speed of $8000 \mathrm{rpm}$. The surface velocities however, varied along the diameter of the workpiece and decreased toward the centre position of the turned workpiece. The depth of cut used in single point diamond turning was much smaller compared with those used in conventional machining. Two levels of depth of cut were used, i.e., 1 and $10 \mu \mathrm{m}$. A feed rate of $20 \mathrm{~mm} / \mathrm{min}$ was used throughout the experiment.

The surface to be machined was chosen to be the (001) plane (Fig. 1). The surface textures before and after cutting were determined by X-ray back diffraction technique on a texture goiniometer (Philip PW3710). The measurement covered the range of $\psi \leq 85^{\circ}$ and $0^{\circ}<\varphi<360^{\circ}$ with the step of $\Delta \psi=\Delta \varphi=3^{\circ} .\{111\}$ pole figures were collected at various locations (A) and (B), which are midway between the centre and the edge of the workpiece as shown in Fig. 1. The diameter of the X-ray irradiated area is adjusted to be around $1 \mathrm{~mm}$ to facilitate the comparison of the differences in textural features at various locations from the turning axis of the workpiece.

\subsection{Analyses of Pole Figures at the Centre}

Figure 2(a)-(c) show the $\{111\}$ pole figures collected at the centre of the turned surface. The $\{111\}$ pole figure of the aluminium single crystal

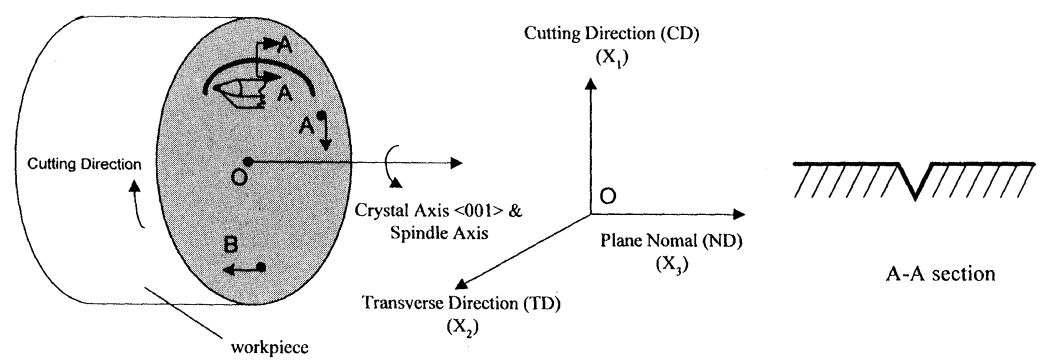

FIGURE 1 Schematic diagram of the diamond turning and the grooving experiment. 
(a)

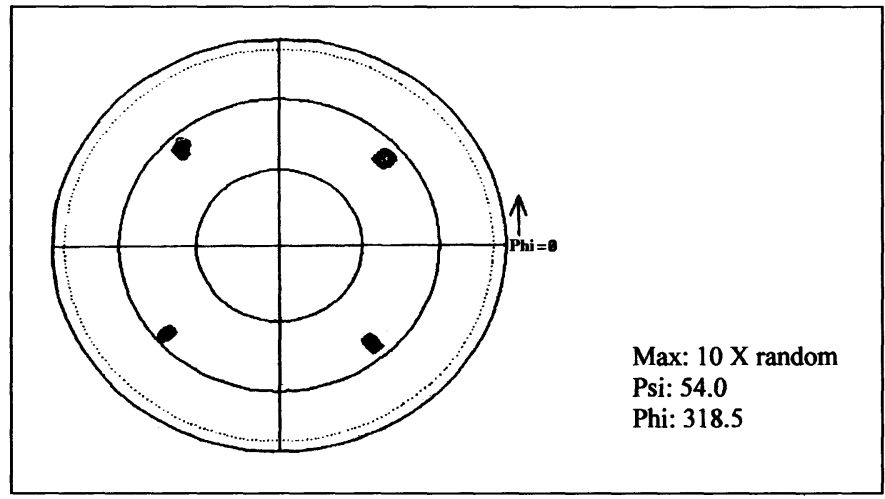

(b)

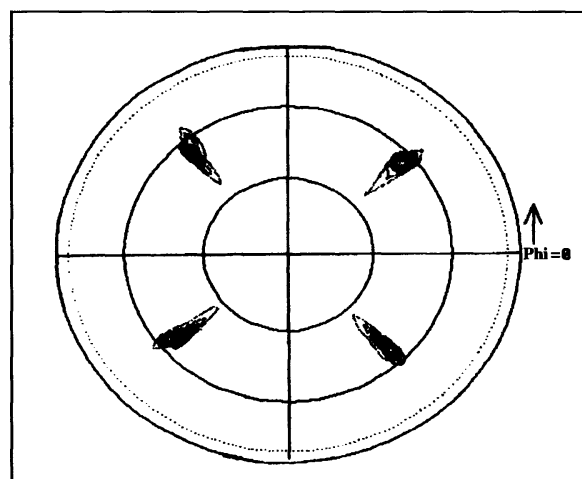

Max: $10 \mathrm{X}$ random

Psi: 51.0

Phi: 228.5

(c)

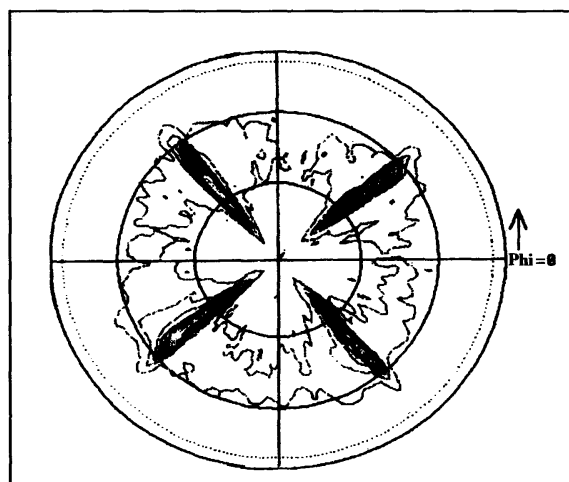

Max: $10.2 \mathrm{X}$ random

Psi: 51.0

Phi: 127.5

FIGURE $2\{111\}$ pole figure of (001) aluminium single crystal in surface layer of centre location (a) before cutting (b) depth of cut $1 \mu \mathrm{m}$ and (c) depth of cut $10 \mu \mathrm{m}$. 
before turning is shown in Fig. 2(a). A typical $\{111\}$ pole figure of a (001) single crystal was recorded which has a four-fold symmetric pattern with respect to the direction normal to the machined surface. Figure 2(b) shows the textural changes after machining at a depth of cut of $1 \mu \mathrm{m}$. All four $\{111\}$ poles tended to rotate towards the centre of the pole figure which indicates a tendency to form a $\{111\}$ texture. As the depth of the X-ray irradiated volume is around $10 \mu \mathrm{m}$, the pole figures were a composite image of both the cutting-induced deformed layer and the undeformed crystal immediately underneath.

To study the textural differences within the deformed layer, about $1 \mu \mathrm{m}$ of the machined surface was removed successively using electrolytic polishing. Two observations were made. Firstly, the extent of lattice rotation was found to decrease as the distance from the machined surface increased. Secondly, the extent of lattice rotation among the poles in the $\{111\}$ pole figure was very similar irrespective of the distance of the exposed layer from the original machined surface. The largest extent of lattice rotation was measured to be around $30^{\circ}$.

With the depth of cut increased to $10 \mu \mathrm{m}$, the effect of cutting-induced textural change is more evident (Fig. 2(c)). The presence of four differently lattice-rotated zones is clearly seen, that resembles the formation of subgrain boundaries in the form of zonal partitioning. Also, as the lattice rotation was induced by cutting action, the partitioning effect on the machined surface is strain-induced. Though the sense of lattice rotation and the morphology of deformation partitioning recorded were very similar to those turned at the depth of cut of $1 \mu \mathrm{m}$, the extent of the lattice rotation was larger and the largest rotation was close to $54^{\circ}$.

The Bragg angles $2 \theta$ were found not to have any significant difference between the uncut specimens and those which have been cut under different depths of cut at various locations on the machined surfaces. The pole figures collected in the present work were based on the same sets of angles, $2 \theta$. This implied that there were no measurable elastic residual strains in the machined layer. The absence of any residual strain at the $\mathrm{X}$-ray level can be associated with the rapid recovery of the high purity aluminium used in the experiment.

The cutting action on the aluminium single crystal induces two kinds of plastic strain onto the machined surface. One is simple shear, $\varepsilon_{13}$ along the cutting direction and the other is a compressive strain, $\varepsilon_{33}$ 
along the direction normal to the machined surface, i.e.,

$$
\begin{aligned}
& \begin{array}{lll}
X_{1} & X_{2} & X_{3}
\end{array} \\
& \left(\begin{array}{ccc}
0.5 \varepsilon_{33} & 0 & \varepsilon_{13} \\
0 & 0.5 \varepsilon_{33} & 0 \\
0 & 0 & -\varepsilon_{33}
\end{array}\right)
\end{aligned}
$$

where $X_{1}$ and $X_{3}$ are directions parallel to the cutting direction (CD) and normal to the machined surface (ND) respectively. $X_{2}$ is the cross product of $X_{3}$ and $X_{1}$ (Fig. 1(b)). Obviously, the shear strain $\varepsilon_{13}$ will induce an asymmetric lattice rotation on the machined surface whereas the normal strain $\varepsilon_{33}$ will not. Since all the pole figures, collected at the centre of the specimens under various depths of cut, have found to be highly symmetric, the magnitude of $\varepsilon_{13}$ is negligible when compared with those of $\varepsilon_{33}$.

As mentioned previously, the dominant mode of deformation strain on the machined surface is $\varepsilon_{33}$. It has been known that crystal lattice tends to rotate under tension with their slip directions or Burgers vectors along the tension direction, and under compression with their normal to the slip plane along the compression axis. Since all the pole figures have shown tendencies towards $\{111\}$ texture, the normal to $\{111\}$ slip planes were aligned parallel to the normal to the machined surface. Judging from the sense of the lattice rotation and the symmetry of the pattern on the pole figures, the imposed strains onto the machined surface were expected to be of the type:

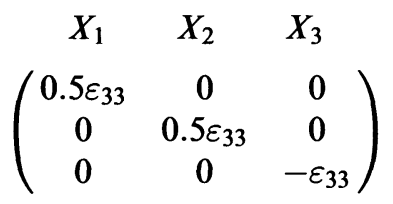

where $X_{1}$ and $X_{3}$ are directions parallel to the cutting direction, CD and to the normal of the machined surface, ND respectively.

\subsection{Analyses of Pole Figures away from the Centre}

Figure 3(a) and (b) shows the pole figures of a [001] aluminium single crystal after diamond turning at a cutting depth of $10 \mu \mathrm{m}$ at two different 
(a)

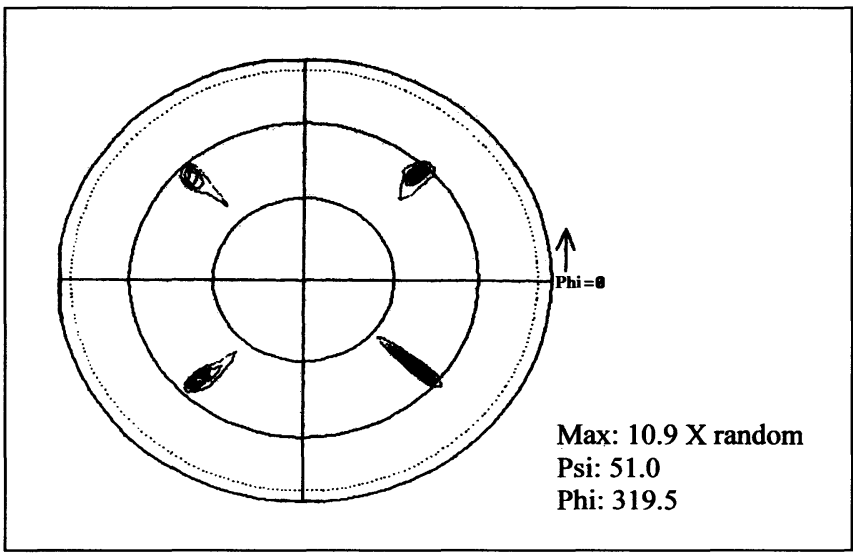

(b)

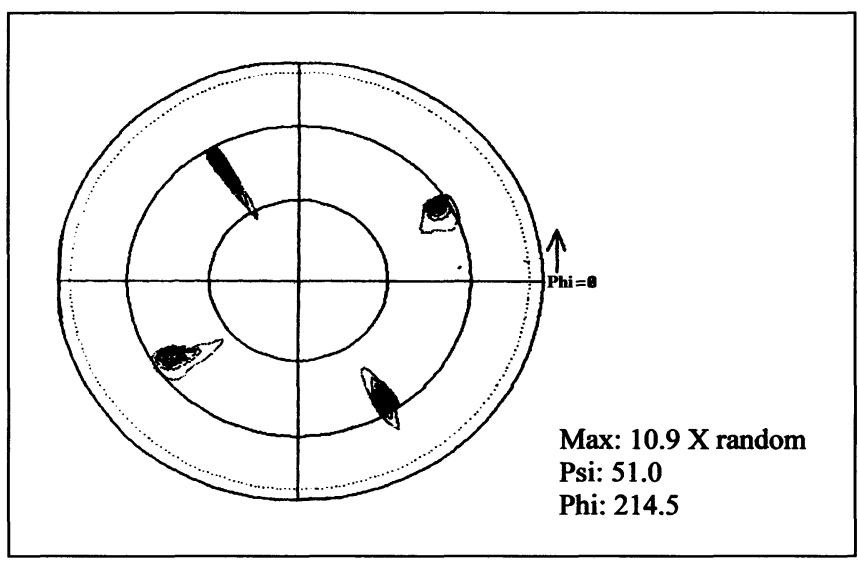

FIGURE $3\{111\}$ texture pole figure of (001) aluminium single crystal after diamond cutting at a cutting depth $10 \mu \mathrm{m}$ in two different positions (A) and (B) as shown in Fig. 1.

locations (A) and (B) (Fig. 1). Three features were noted when compared with those collected at the centre of the specimens:

(i) The compression texture is less stronger.

(ii) The extent of deformation among the four $\{111\}$ poles is asymmetric.

(iii) The sense of lattice rotation is affected by the cutting direction. 
TABLE I Effect of cutting velocity on the surface texture

\begin{tabular}{lll}
\hline Cutting velocity & $\begin{array}{c}\text { Compression } \\
\text { texture }\end{array}$ & Lattice rotation \\
\hline $\begin{array}{l}\text { Low (near the centre) } \\
\left(8 \times 10^{3} \mathrm{~mm} / \mathrm{min}\right)\end{array}$ & Strong & Symmetric \\
$\begin{array}{c}\text { High }(\text { at the rim }) \\
\left(3.2 \times 10^{5} \mathrm{~mm} / \mathrm{min}\right)\end{array}$ & Weak & $\begin{array}{l}\text { Asymmetric and depends on } \\
\text { the cutting direction }\end{array}$ \\
\hline
\end{tabular}

The plastic strain on the machined surface is expected to be approximated by:

$$
\begin{gathered}
X_{1} X_{2} \\
X_{3} \\
\left(\begin{array}{ccc}
0 & 0 & \varepsilon_{13} \\
0 & 0 & 0 \\
0 & 0 & 0
\end{array}\right)
\end{gathered}
$$

where $X_{1}$ and $X_{3}$ are directions parallel to CD and to ND respectively. In terms of the cutting conditions, the major differences between the centre and at locations away from the centre lies in the difference cutting velocity.

The turning speed used in the present work is $8000 \mathrm{rpm}$ and the cutting velocity at a particular location can be obtained from the turning speed and the distance from the centre. Thus, the cutting speed is the lowest at the centre while the highest at the rim of the machined surface. Based on this observation, it is suggested that the cutting velocity has a strong effect on the finished texture and is summarised in Table I. A strong compression texture with symmetric lattice rotation is associated with low machining speed. On the other hand, a weak compression texture is found at workpiece turned at high cutting speed.

\section{PART 2. ELECTRON BACK-SCATTER DIFFRACTION STUDIES}

\subsection{Experimental Procedure}

Another cutting experiment was performed on a two axis CNC contouring lathe from Taylor Hobson Precision (Optoform 30) with the same workpiece material and cutting tool. Prior to machining, the 
workpiece was electropolished to remove any surface deformed layer. Electropolishing was carried out in a 10:90 mixture of $70 \% \mathrm{HClO}_{4}$ and $\mathrm{C}_{2} \mathrm{H}_{5} \mathrm{OH}$, and at a voltage of $30 \mathrm{~V}$.

A circular groove was produced on the (001) plane of the aluminium single crystal by rotating the crystal $40^{\circ}$ in an anti-clockwise direction on the lathe (Fig. 1). The depth of cut was $250 \mu \mathrm{m}$. EBSD patterns were collected along the bottom part of the groove (Fig. 4) to study the lattice rotation induced by machining. Electrolytic polishing was used to remove the deformed surface on the substrate layer. Approximately a layer of $25 \mu \mathrm{m}$ thick was removed each time. EBSD patterns were

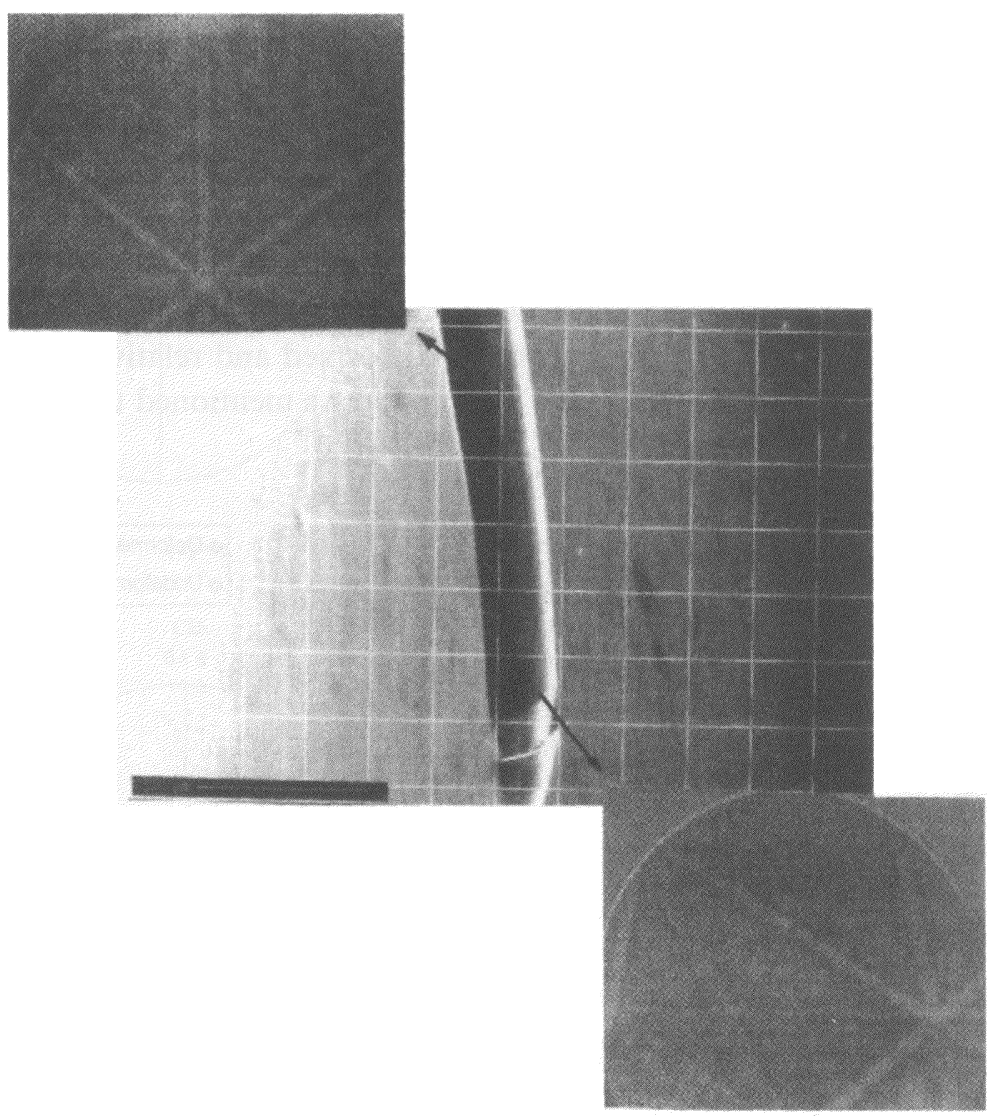

FIGURE 4 Turned groove on (001) aluminium single crystal. 
collected repeatedly at various locations along the freshly electrolytic polished groove. The orientation changes were then determined by EBSD technique.

\subsection{EBSD Studies}

After machining, there were surface areas which were affected (i.e., deformed) and areas which were relatively unaffected (i.e., undeformed) as indicated by the rotation of the plane normal of the (001) crystal surface. Some of the plane normals of the surface were found to rotate away from the initial [001] direction about the [100] rotation axis of the crystal. Such rotation did, however, not occur all the way along the machined groove surface but was found to be of intermittent nature. When rotation did occur, it tended to rotate $65^{\circ}$ away from the [001] towards the [112] direction. A plot of the lattice rotation along the groove is shown in Fig. 4.

By successive removal of the surface metal on the groove by electropolishing, about twenty EBSP patterns were collected along the groove at various depths below the machined surface. Figure 5 shows the derivation of the plane normals of the deformed and relatively undeformed areas on the metal layer from (001). As mentioned there were

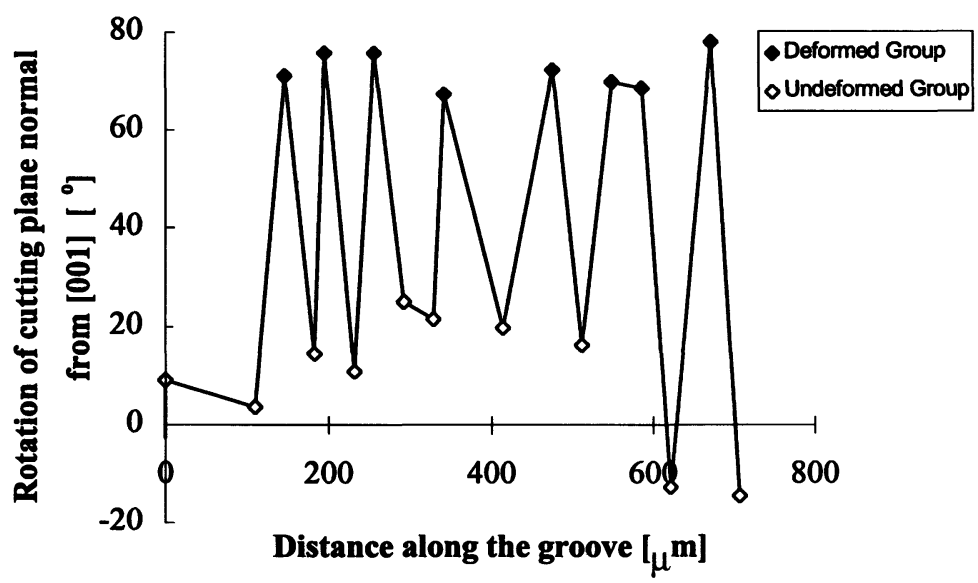

FIGURE 5 Distribution of lattice rotation of cutting plane normal from [001] along the groove. 
two main groups of orientations. The plane normals were clustered about either [112] direction or [001] direction, the relative proportion of which would indicate the proportion of the areas which were affected by the cutting tool and those which were not affected. At 50 and $100 \mu \mathrm{m}$ below the groove surface, there was no significant decrease in the deformed area (Fig. 6(b) and (c)). However at $150 \mu \mathrm{m}$ below the groove surface, the fraction of the deformed area decreased sharply (Fig. 6(d)). At about $250-300 \mu \mathrm{m}$ below the groove surface, the deformed area disappeared completely.

The cutting direction at various locations on the machined groove was parallel to the tangent at that locations. However, as the groove

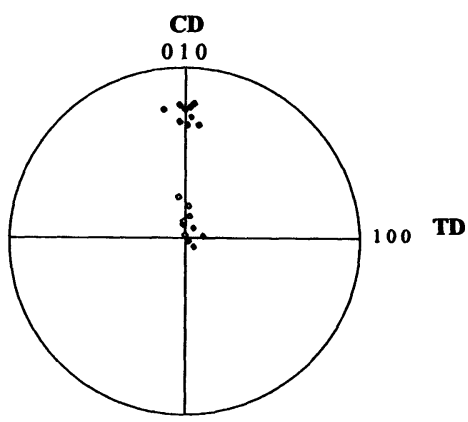

(a)

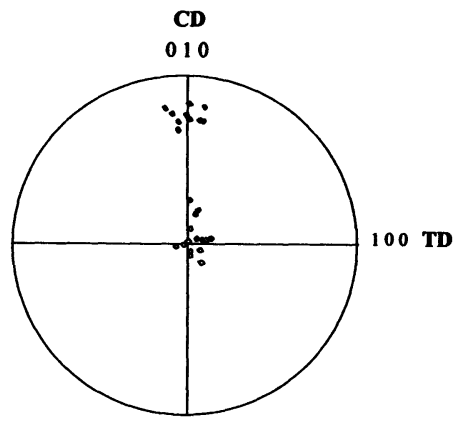

(c)

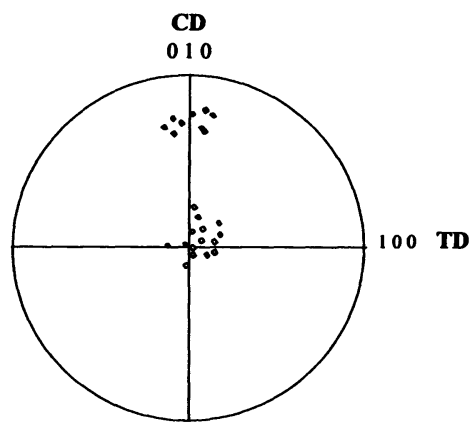

(b)

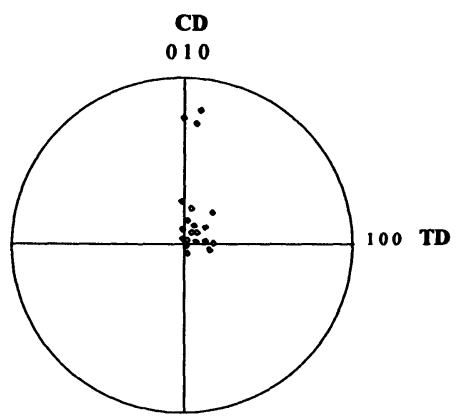

(d)

FIGURE 6 Stereographic projection of orientation distributions of the machined layer at (a) $0 \mu \mathrm{m}$ (b) $50 \mu \mathrm{m}$ (c) $100 \mu \mathrm{m}$ and (d) $150 \mu \mathrm{m}$ along the groove. 
was relatively short which spanned an angle of around $40^{\circ}$, the cutting direction could be taken to be along [010] direction. Based on the experimental findings, the cutting plane normal tends to rotate from [001] towards [112]. Judging from the fact that there was a $60-65^{\circ}$ lattice rotation on the machined surface, large plastic shear was expected to have been induced. An estimated shear strain of about 2.5 was needed to generate such a large shear rotation, and this observation is in line with the prediction that there is a tendency for [001] cutting plane normal to rotate towards [112] direction upon shearing.

\section{CONCLUSIONS}

Single point diamond turning induces plastic strain on the machined surface that is very dependent on the cutting velocity. Low cutting velocity will result in the formation of a stronger compression texture which involves deformation partitioning of four equally strained slip systems. High cutting velocity would induce an intense shear on the machined surface on which the sense of shear is dependent on the cutting direction and so is the lattice rotation induced.

A significant finding from this experiment is that the shearing of the surface layer induced by the cutting tool was of intermittent nature, i.e., there were surface areas which were disturbed and areas which were not. In conventional machining, the entire machined surface is plastically deformed and stress-free area is rare. This could be partly due to the fact that there is some extrusion of the surface layer material beneath the cutting tool with a certain radius. In the experiment reported in this paper, both the depth of cut and the tool radius are an order of magnitude lower than those values used in conventional cutting. The effect of the extrusion would be smaller under the single point diamond turning condition.

\section{Acknowledgements}

The authors wish to thank the Research Committee of The Hong Kong Polytechnic University for the support of a research grant on this investigation work. 


\section{References}

König, W. and Senrath, N. (1991) Progression Precision Engineering, Springer-Verlag, Braunschweig, Germany, p. 141.

Krause, H. and Öcalan, E. (1984) Proceedings of the Seventh International Conference on Texture of Materials, Eds. C.M. Brakman, P. Jongenburger and E.J. Mittmeijer, p. 625.

Lee, W.B. (1990) Precision Engineering, 12, 1, 25.

Lee, W.B., To, S. and Chan, C.Y. (1996) Proceedings of Eleventh International Conference on Texture of Materials, Xian, China, 16-20 September, p. 293.

Lee, W.B. and Zhou, M. (1993) International Journal of Machine Tool and Manufacture, 33, 439.

Maurer, G., Neff, H., Scholtes, B. and Macherauch, E. (1988) Textures and Microstructures, 8 \& 9, 639.

Moriwaki, T., Okuda, K. and Shen, J.G. (1993) JSME International Journal, Series C, 36, 400.

Ueda, K. and Iwata, K. (1980) Annals of the CIRP, 29, 41.

Yuan, Z.J., He, J.C. and Yao, Y.X. (1992) Annals of the CIRP, 41, 65.

Yuan, Z.J., Lee, W.B., Yao, Y.X. and Zhou, M. (1994) Annals of the CIRP, 43, 39. 